\title{
Administration of recombinant tissue plasminogen activator to a case of cerebral infarction in the setting of painless aortic dissection
}

Sir,

A 72-year-old female presented with acute onset left sided hemiparesis. On admission, a physical examination revealed a consciousness level of 15 on the Glasgow coma scale, blood pressure of $160 / 116 \mathrm{mmHg}$, and pulse rate of 51 per minute. 

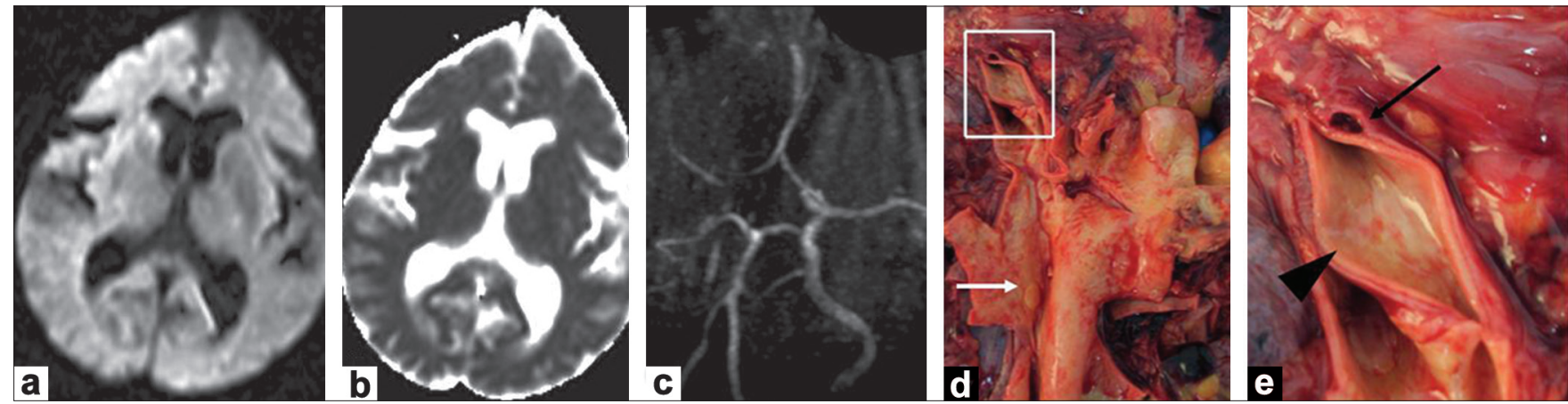

Figure 1: (a,b) Diffusion-weighted image reveals elevated diffusion coefficients with high signal intensity in the right middle cerebral artery territory. Apparent diffusion coefficient maps are decreased. (c) Brain magnetic resonance angiography demonstrates an occlusion of the right carotid artery $(\mathrm{d}, \mathrm{e})$ Photograph taken at autopsy reveals a Stanford Type A aortic dissection and cardiac tamponade. Dissection is also seen in the right common carotid artery. The boxed area in $d$ is shown at higher magnification in e. White arrow - Ascending aorta, black arrowhead - Right CCA, black arrow False lumen

Radial and femoral pulses were normal. Patient had left hmeparesis and National Institutes of Health Stroke Scale (NIHSS) score was 16. She did not complain any chest, abdominal and back pain. Chest X-ray, an electrocardiogram and brain computed tomography (CT) showed no abnormal findings. Brain magnetic resonance imaging (MRI) revealed a right middle cerebral artery territory infarct [Figure 1a and b]. Brain MR angiography demonstrated an occlusion of right carotid artery [Figure 1c]. Laboratory studies were within normal limits. One hundred and fortyfour min after onset, the patient received recombinant tissue plasminogen activator (rt-PA), $39 \mathrm{mg}(0.6 \mathrm{mg} /$ $\mathrm{kg}$ ). On Day 2, vital signs were stable and follow-up CT showed no hemorrhagic change. However, on Day 3, the patient suffered a sudden cardiac arrest from which she could not be resuscitated. Autopsy revealed a Stanford Type A aortic dissection (AD) and cardiac tamponade [Figure 1d]. There was also dissection of the right common carotid artery [Figure $1 \mathrm{~d}$ and e].

Thrombolysis with rt-PA has been done in the setting of painless AD. ${ }^{[1]}$ In our patient it is quite possible that the administration of rt-PA might have contributed to the death of the patient by the lysis of the thrombus that would otherwise have limited the dissection. Because AD is a rare cause of stroke, there is debate about the necessity to aggressively screen for AD.

Although pain in the chest, back, or abdomen is a common symptom in AD, approximately $10 \%$ of patients experience no pain. ${ }^{[2-5]}$ Pulse and blood pressure differences and abnormal chest radiograms can be useful for the rapid assessment of AD. However, approximately $7 \%$ of AD patients show normal findings. ${ }^{[2]}$ Color duplex ultrasound is a simple tool to exclude painless AD and cervical artery dissection. ${ }^{[3,4]}$ Chest CT scanning and transesophageal echocardiography, while very sensitive for $\mathrm{AD}$, are time-consuming and expensive. ${ }^{[2-4]}$

Strokes associated with $\mathrm{AD}$ are predominantly right hemispheric..$^{[5]}$ This dominance could be explained by varying mechanical dynamics in the progression of the dissecting hematoma. We believe a high index of suspicion of painless AD would be advisable and also a lower threshold for performing carotid ultrasound before rt-PA treatment, especially in patients with right hemispheric infarct.

\section{Satoru Takeuchi, Yoshio Takasato, Hiroyuki Masaoka, Naoki Otani}

Department of Neurosurgery, National Hospital Organization Disaster Medical Center, Japan. E-mail: s.takeuchi@room.ocn.ne.jp

DOI: $10.4103 / 0028-3886.59487$

\section{References}

1. Fessler AJ, Alberts MJ. Stroke treatment with tissue plasminogen activator in the setting of aortic dissection. Neurology 2000;54:1010.

2. von Kodolitsch Y, Schwartz AG, Nienaber CA. Clinical prediction of acute aortic dissection. Arch Intern Med 2000 23;160:2977-82.

3. Veyssier-Belot C, Cohen A, Rougemont D, Levy C, Amarenco P, Bousser MG. Cerebral infarction due to painless thoracic aortic and common carotid artery dissections. Stroke 1993;24:2111-3.

4. Benninger DH, Georgiadis D, Gandjour J, Baumgartner RW. Accuracy of color duplex ultrasound diagnosis of spontaneous carotid dissection causing ischemia. Stroke 2006;37:377-81.

5. Gaul C, Dietrich W, Friedrich I, Sirch J, Erbguth FJ. Neurological symptoms in type A aortic dissections. Stroke 2007;38:292-7.

Accepted on 12-05-2009 